\title{
A THEOREM ON DERIVATIONS ON PRIME RINGS
}

\author{
KUN-SHAN LIU \\ (Received 22 June 2010; accepted 15 June 2011)
}

Communicated by B. J. Gardner

\begin{abstract}
Let $R$ be a prime ring, let $I$ be a nonzero ideal of $R$ and let $n$ be a fixed positive integer. We prove that if the characteristic of $R$ is either 0 or a prime $p$ that is greater than $2 n$, then an additive map $d$ that satisfies $d\left(x^{n+1}\right)=\sum_{j=0}^{n} x^{n-j} d(x) x^{j}$ for all $x \in I$ must be a derivation.

2010 Mathematics subject classification: primary 16W25; secondary 16N60, 16R20, 16R60.

Keywords and phrases: prime ring, derivations, additive map, generalized polynomial identity, functional identity.
\end{abstract}

\section{Introduction}

This paper is about functional identities in ring theories. We study an additive map that satisfies a specific identity and generalize some results about derivations on prime rings.

Throughout this paper, $R$ is always a prime ring, not necessarily with identity, which has center $Z(R)$. Let $Q_{r}(R)$ be the right Martindale quotient ring of $R$; this is a ring characterized by the following axioms (see [3, Proposition 2.2.1]).

(1) The ring $R$ is a subring of $Q_{r}(R)$.

(2) For each $a \in Q_{r}(R)$, there exists a nonzero ideal $I$ of $R$ such that $a I \subseteq R$.

(3) If $a \in Q_{r}(R)$ and $a I=0$ for some nonzero ideal $I$ of $R$, then $a=0$.

(4) For any ideal $I$ of $R$ and any right $R$-module map $\phi: I_{R} \rightarrow R_{R}$, there exists $a \in Q_{r}(R)$ such that $\phi(r)=a r$ for all $r \in I$.

Let $Q(R)$ denote the set of all $a \in Q_{r}(R)$ such that $J a \subseteq R$ for some nonzero $J \triangleleft R$. Then $Q(R)$ is called the symmetric Martindale quotient ring of $R$.

The overrings $Q_{r}(R)$ and $Q(R)$ are also prime rings. The center $C$ of $Q(R)$ is a field, called the extended centroid of $R$. The ring $R C$ is called the central closure of $R$. We refer to [3] for more details.

This work was supported by National Taiwan University under Grant 99R40044.

(C) 2011 Australian Mathematical Publishing Association Inc. 1446-7887/2011 \$16.00 
For $a, b \in R$, we denote by $[a, b]$ the commutator $a b-b a$ of $a$ and $b$. For two additive subgroups $A$ and $B$ of $R$, we denote by $[A, B]$ the additive subgroup of $R$ generated by all elements $[a, b]$ for $a \in A$ and $b \in B$. An additive subgroup $L$ of $R$ is called a Lie ideal if $[L, R] \subseteq L$.

A polynomial identity (PI) of $R$ is defined to be a polynomial $f\left(X_{1}, X_{2}, \ldots, X_{n}\right)$ in noncommutative variables with coefficients in $C$ such that $f\left(X_{1}, X_{2}, \ldots, X_{n}\right)$ vanishes for all substitutions of the $X_{i}$ by elements of $R$. A ring is called a PI-ring if it satisfies a nontrivial polynomial identity. For example, any commutative ring satisfies the identity $\left[X_{1}, X_{2}\right]$. More generally, a generalized polynomial of $R$ is a polynomial in noncommutative variables with coefficients in $R C$, that is, an element in the free product

$$
R C\left\{X_{1}, X_{2}, \ldots, X_{n}\right\}=R C *_{C} C\left\{X_{1}, X_{2}, \ldots, X_{n}\right\} .
$$

A ring is called a GPI-ring if it satisfies a nontrivial generalized polynomial identity. See $[3,13]$ for more details.

Suppose that $I$ is an ideal of $R$. An additive map $d: I \rightarrow R$ is said to be a derivation on $I$ if $d(x y)=d(x) y+x d(y)$ for all $x, y \in I$. For example,

$$
d(x)=[a, x]=a x-x a,
$$

where $a \in R$ is a derivation on $I$. A derivation of this form is called an inner derivation. An additive map $d: I \rightarrow R$ is called a Jordan derivation if

$$
d\left(x^{2}\right)=d(x) x+x d(x) \quad \forall x \in I .
$$

Any derivation must be a Jordan derivation, but the converse is not true in general.

In 1957, Herstein [11, Theorem 3.1] proved that a Jordan derivation on a prime ring of characteristic other than 2 must be a derivation. In 1975, Cusack [9, Corollary 5] generalized Herstein's result to a 2-torsion free semiprime ring. In 1988, Brešar and Vukman [6] gave a brief proof for Herstein's result and Brešar [4] also gave an alternative proof for Cusack's result.

More generally, if $d$ is a derivation on $R$, then

$$
d\left(x^{n}\right)=\sum_{j=1}^{n} x^{n-j} d(x) x^{j-1} \quad \forall x \in R,
$$

but the converse is not true in general. Bridges and Bergen [7, Theorem 2] proved that the converse is true if $R$ is an $n$ !-torsion free prime ring with identity. Vukman and Kosi-Ulbl [18, Theorem 1] generalized this result to an $n$ !-torsion free semiprime ring with identity. They also asked whether we can prove the theorem without assuming that $R$ has an identity, but with a suitable torsion restriction.

In this paper, we will focus on the prime case and just assume that $d$ is defined on a nonzero ideal of $R$. More precisely, our main theorem is the following result. 
THEOREM 1.1. Let $R$ be a prime ring, let $I$ be a nonzero ideal of $R$ and let $n$ be a fixed positive integer. Suppose that $\operatorname{char}(R)$, the characteristic of $R$, is either 0 or a prime $p$ that is greater than $2 n$. If $d: I \rightarrow R$ is an additive map such that

$$
d\left(x^{n+1}\right)=\sum_{j=0}^{n} x^{n-j} d(x) x^{j}
$$

for all $x \in I$, then $d$ is a derivation on $I$.

We note that Beidar et al. [2] investigated a special functional identity that is related to Theorem 1.1. In fact, they proved the following result [2, Theorem 4.4]. Let $R$ be a prime ring such that $2 \neq \operatorname{char}(R) \nmid n$. Suppose that $I$ and $R$ are centrally closed, that is, $I C=I$ and $R C=R$. If $d$ is a $C$-linear map such that $d\left(x^{n+1}\right)=\sum_{j=0}^{n} x^{n-j} d(x) x^{j}$ for all $x \in I$, then $d$ is a derivation on $I$.

Essentially, a $C$-linear derivation that is algebraic over $C$ must be an inner derivation of $Q(R)$ (see [15, Corollary 2]). But, any derivation clearly satisfies the expansion formula in Theorem 1.1. In other words, by [2, Theorem 4.4] the $C$-linear case can be proved under a weaker restriction on $\operatorname{char}(R)$. In this paper, although we have a stronger restriction on $\operatorname{char}(R)$, we prove that $d$ is a derivation in a general case. Moreover, we do not need to assume that $I$ is centrally closed, but we will prove that the map $d$ on $I$ can be extended to a map $\tilde{d}$ on $R C$ when $R$ is a PI-ring.

Recently, Fošner and Vukman investigated a similar identity. They proved that in a prime ring $R$ such that $2 \neq \operatorname{char}(R) \geq 2 n$ an additive map $T: R \rightarrow R$ satisfying

$$
T\left(x^{n+1}\right)=\sum_{j=0}^{n}(-1)^{j+1} x^{n-j} T(x) x^{j}
$$

must be of the form $4 T(x)=q x+x q$ for some $q \in Q(R)$ (see [10, Theorem 3]).

\section{Proofs}

We always assume that $R$ is a prime ring and the characteristic of $R$ is either 0 or a prime $p$ that is greater than $2 n$, where $n$ is a fixed positive integer. We will use our assumption on the characteristic without further explanation to solve equations by the van der Monde argument and to cancel some invertible integers.

We shall prove Theorem 1.1 using a sequence of lemmas. For $x, y \in R$, we denote by $S_{x, y}(k, s-k)$ the sum of all monic monomials with $k$ occurrences of $x$ and $s-k$ occurrences of $y$. For example,

$$
S_{x, y}(2,1)=x^{2} y+x y x+y x^{2} .
$$

First, we prove that $R$ satisfies a specific functional identity. 
LeMma 2.1. Let $d: I \rightarrow R$ be an additive map, where $I$ is a nonzero ideal of $R$. Suppose that $d\left(x^{n+1}\right)=\sum_{j=0}^{n} x^{n-j} d(x) x^{j}$ for all $x \in I$. Then

$$
\sum_{j=0}^{n}\left((n-j) x^{2 n-2 j-1} T(x, x) x^{2 j}+(n-j) x^{2 j} T(x, x) x^{2 n-2 j-1}\right)=0
$$

for all $x \in I$, where

$$
T(x, y)=\frac{1}{2}(d(x y)-d(x) y-x d(y)+d(y x)-d(y) x-y d(x))
$$

is a symmetric biadditive map.

Proof. Let $x, y \in I$. Expanding

$$
d\left((x+y)^{n+1}\right)=\sum_{j=0}^{n}(x+y)^{n-j} d(x+y)(x+y)^{j}
$$

and using the identities

$$
d\left(x^{n+1}\right)=\sum_{j=0}^{n} x^{n-j} d(x) x^{j}
$$

and

$$
d\left(y^{n+1}\right)=\sum_{j=0}^{n} y^{n-j} d(y) y^{j}
$$

we see that

$$
\begin{aligned}
\sum_{k=1}^{n} d\left(S_{x, y}(n+1-k, k)\right)=\sum_{j=0}^{n} & \left(x^{n-j} d(x+y) \sum_{k=1}^{j} S_{x, y}(j-k, k)\right) \\
& +\sum_{j=0}^{n}\left(y^{n-j} d(x+y) \sum_{k=0}^{j-1} S_{x, y}(j-k, k)\right) \\
& +\sum_{j=0}^{n}\left(\left(\sum_{k=1}^{j-1} S_{x, y}(j-k, k)\right) d(x+y)\right. \\
& \left.\times\left(\sum_{k=0}^{j} S_{x, y}(j-k, k)\right)\right) .
\end{aligned}
$$

Since either $\operatorname{char}(R)=0$ or $\operatorname{char}(R)=p>2 n>1$, we will obtain $n$ equations by replacing $y$ by $y, 2 y, \ldots, n y$ in (2.2) in turn. Then, applying the van der Monde argument to solve these $n$ equations, we see that

$$
\begin{aligned}
d\left(S_{x, y}(n, 1)\right)=\sum_{j=0}^{n} & \left(S_{x, y}(n-j-1,1) d(x) x^{j}\right. \\
& \left.+x^{n-j} d(y) x^{j}+x^{n-j} d(x) S_{x, y}(j-1,1)\right) .
\end{aligned}
$$


Replacing $x$ by $x^{2}$ and $y$ by $x$ in (2.3) yields

$$
\begin{aligned}
(n+1) d\left(x^{2 n+1}\right)=\sum_{j=0}^{n} & \left((n-j) x^{2 n-2 j-1} d\left(x^{2}\right) x^{2 j}\right. \\
& \left.+x^{2 n-2 j} d(x) x^{2 j}+j x^{2 n-2 j} d\left(x^{2}\right) x^{2 j-1}\right) .
\end{aligned}
$$

On the other hand, replacing $y$ by $x^{n+1}$ (leaving $x$ unchanged) in (2.3), we see that

$$
\begin{aligned}
(n+1) d\left(x^{2 n+1}\right) & =\sum_{j=0}^{n}\left((n-j) x^{2 n-j} d(x) x^{j}+x^{n-j} d\left(x^{n+1}\right) x^{j}+j x^{n-j} d(x) x^{n+j}\right) \\
& =\sum_{j=0}^{n}\left((n-j) x^{2 n-j} d(x) x^{j}+\sum_{i=0}^{n} x^{2 n-i-j} d(x) x^{i+j}+j x^{n-j} d(x) x^{n+j}\right) .
\end{aligned}
$$

Combining (2.4) and (2.5), we see that

$$
\begin{aligned}
\sum_{j=0}^{n}( & (n-j) x^{2 n-2 j-1}\left(d\left(x^{2}\right)-d(x) x-x d(x)\right) x^{2 j} \\
+ & \left.(n-j) x^{2 j}\left(d\left(x^{2}\right)-d(x) x-x d(x)\right) x^{2 n-2 j-1}\right) \\
= & \sum_{j=0}^{n}\left((n-j) x^{2 n-j} d(x) x^{j}+(n-j) x^{j} d(x) x^{2 n-j}+\sum_{i=0}^{n} x^{2 n-i-j} d(x) x^{i+j}\right. \\
& -(n-j) x^{2 n-2 j} d(x) x^{2 j}-(n-j) x^{2 j} d(x) x^{2 n-2 j}-x^{2 n-2 j} d(x) x^{2 j} \\
& \left.-(n-j) x^{2 n-2 j-1} d(x) x^{2 j+1}-(n-j) x^{2 j+1} d(x) x^{2 n-2 j-1}\right) .
\end{aligned}
$$

We perform some complicated and tricky computations with the summations to show that the sum on the right-hand side is zero.

First, we compute the sum of the first three terms:

$$
\begin{aligned}
\sum_{j=0}^{n}(n-j) x^{2 n-j} d(x) x^{j}+\sum_{j=0}^{n}(n-j) x^{j} d(x) x^{2 n-j}+\sum_{j=0}^{n} \sum_{i=0}^{n} x^{2 n-i-j} d(x) x^{i+j} \\
=\sum_{j=0}^{n}(n-j) x^{2 n-j} d(x) x^{j}+\sum_{j=0}^{n-1}(n-j) x^{j} d(x) x^{2 n-j} \\
\quad+\sum_{j=0}^{n}(j+1) x^{2 n-j} d(x) x^{j}+\sum_{j=0}^{n-1}(j+1) x^{j} d(x) x^{2 n-j} \\
=\sum_{j=0}^{n}(n+1) x^{2 n-j} d(x) x^{j}+\sum_{j=0}^{n-1}(n+1) x^{j} d(x) x^{2 n-j} \\
=(n+1) \sum_{j=0}^{2 n} x^{2 n-j} d(x) x^{j} .
\end{aligned}
$$


Next, we compute the sum of second three terms:

$$
\begin{aligned}
\sum_{j=0}^{n}(( & \left.n-j) x^{2 n-2 j} d(x) x^{2 j}+(n-j) x^{2 j} d(x) x^{2 n-2 j}+x^{2 n-2 j} d(x) x^{2 j}\right) \\
& =\sum_{j=0}^{n}\left(j x^{2 j} d(x) x^{2 n-2 j}+(n-j) x^{2 j} d(x) x^{2 n-2 j}+x^{2 j} d(x) x^{2 n-2 j}\right) \\
& =\sum_{j=0}^{n}(n+1) x^{2 j} d(x) x^{2 n-2 j}
\end{aligned}
$$

Finally, the sum of the last two terms is

$$
\begin{aligned}
& \sum_{j=0}^{n}\left(j x^{2 j-1} d(x) x^{2 n-2 j+1}\right)+\sum_{j=0}^{n}\left((n-j) x^{2 j+1} d(x) x^{2 n-2 j-1}\right) \\
& =\sum_{j=0}^{n-1}\left((j+1) x^{2 j+1} d(x) x^{2 n-2 j-1}\right)+\sum_{j=0}^{n-1}\left((n-j) x^{2 j+1} d(x) x^{2 n-2 j-1}\right) \\
& =\sum_{j=0}^{n-1}(n+1) x^{2 j+1} d(x) x^{2 n-2 j-1}
\end{aligned}
$$

Substituting (2.7), (2.8) and (2.9) into (2.6) enables us to see that the right-hand side of (2.6) is equal to

$$
\begin{aligned}
& (n+1) \sum_{j=0}^{2 n} x^{2 n-j} d(x) x^{j}-\sum_{j=0}^{n}(n+1) x^{2 j} d(x) x^{2 n-2 j}-\sum_{j=0}^{n-1}(n+1) x^{2 j+1} d(x) x^{2 n-2 j-1} \\
& =(n+1) \sum_{j=0}^{2 n} x^{2 n-j} d(x) x^{j}-(n+1) \sum_{j=0}^{2 n} x^{2 n-j} d(x) x^{j}=0
\end{aligned}
$$

as asserted. Hence, by (2.6),

$$
\begin{aligned}
& \sum_{j=0}^{n}\left((n-j) x^{2 n-2 j-1}\left(d\left(x^{2}\right)-d(x) x-x d(x)\right) x^{2 j}\right. \\
& \left.\quad+(n-j) x^{2 j}\left(d\left(x^{2}\right)-d(x) x-x d(x)\right) x^{2 n-2 j-1}\right)=0
\end{aligned}
$$

for all $x \in I$. This completes the proof.

Following Lemma 2.1, we can prove that the image of a central element in $R$ under the map $d$ is still central.

LEMma 2.2. Under the assumptions of Lemma 2.1, if $c \in I \cap Z(R)$, then $d(c) \in Z(R)$ and $d(c x)=d(c) x+c d(x)$ for all $x \in I$. 
Proof. Replacing $x$ by $c$ in (2.3), we see that

$$
\begin{aligned}
(n+1) d\left(c^{n} y\right) & =\sum_{j=0}^{n}\left((n-j) c^{n-1} y d(c)+c^{n} d(y)+j c^{n-1} d(c) y\right) \\
& =2^{-1} n(n+1) c^{n-1} y d(c)+(n+1) c^{n} d(y)+2^{-1} n(n+1) c^{n-1} d(c) y .
\end{aligned}
$$

Replacing $y$ by $y^{n+1}$ in (2.10), we see that

$$
\begin{array}{rl}
(n+1) d\left(c^{n} y^{n+1}\right)=2^{-1} & n(n+1) c^{n-1} y^{n+1} d(c) \\
& +(n+1) c^{n} d\left(y^{n+1}\right)+2^{-1} n(n+1) c^{n-1} d(c) y^{n+1} \\
= & 2^{-1} n(n+1) c^{n-1} y^{n+1} d(c)+(n+1) c^{n} \sum_{j=0}^{n} y^{n-j} d(y) y^{j} \\
& +2^{-1} n(n+1) c^{n-1} d(c) y^{n+1} .
\end{array}
$$

On the other hand, replacing $x$ by $y$ and $y$ by $c^{n} y$ in (2.3) and applying (2.10), we see that

$$
\begin{aligned}
(n+1) d\left(c^{n} y^{n+1}\right)= & \sum_{j=0}^{n}\left((n-j) c^{n} y^{n-j} d(y) y^{j}+y^{n-j} d\left(c^{n} y\right) y^{j}\right. \\
& \left.+j c^{n} y^{n-j} d(y) y^{j}\right) \\
= & \sum_{j=0}^{n}\left(n c^{n} y^{n-j} d(y) y^{j}+2^{-1} n c^{n-1} y^{n-j+1} d(c) y^{j}\right. \\
& \left.+c^{n} y^{n-j} d(y) y^{j}+2^{-1} n c^{n-1} y^{n-j} d(c) y^{j+1}\right) \\
= & \sum_{j=0}^{n}\left(2^{-1} n c^{n-1} y^{n-j+1} d(c) y^{j}+(n+1) c^{n} y^{n-j} d(y) y^{j}\right. \\
& \left.+2^{-1} n c^{n-1} y^{n-j} d(c) y^{j+1}\right) .
\end{aligned}
$$

From (2.11) and (2.12), we deduce that

$$
2 c^{n-1} \sum_{j=1}^{n} y^{n-j+1} d(c) y^{j}=n c^{n-1} y^{n+1} d(c)+n c^{n-1} d(c) y^{n+1}
$$

for all $y \in I$.

Suppose that $d(c)$ is not in $Z(R)$. Then (2.13) implies that $R$ is a prime GPI-ring. By [17, Theorem 3], $Q(R)$ possesses nontrivial idempotents. By [3, Theorem 6.4.1] or [8, Theorem 2], (2.13) holds for all $y \in Q(R)$. So, if we replace $y$ by an idempotent $e$ in (2.13), then we see that

$$
n e d(c)=(2 n-1) e d(c) e=n d(c) e
$$

and hence $[d(c), e]=0$ for all idempotents in $Q(R)$. 
Let $E$ denote the additive subgroup of $Q(R)$ generated by all idempotents and let $\bar{E}$ denote the subring of $Q(R)$ generated by $E$. Then $[d(c), \bar{E}]=0$. It is well known that $E$ is a Lie ideal of $Q(R)$. Since there exists a nontrivial idempotent $e$ in $Q(R)$ such that $[e, e x(1-e)] \neq 0$ for some $x \in Q(R)$, we know that $[E, E] \neq 0$. Moreover, by [12, Lemma 1.3], $Q(R)[E, E] Q(R) \subseteq \bar{E}$. So, $d(c)$ commutes with a nonzero ideal of $Q(R)$ and this forces $d(c) \in C$, a contradiction. Thus, $d(c) \in Z(R)$ for all $c \in I \cap Z(R)$.

Replacing $x$ by $c$ in (2.1) shows that $d\left(c^{2}\right)=2 c d(c)$. Replacing $x$ by $x+c$, $x+2 c, \ldots, x+(m+n) c$ in (2.1), applying the van der Monde argument to solve these $m+n$ equations and using $d\left(c^{2}\right)=2 c d(c)$ allows us to deduce that

$$
2 d(c x)=2 d(x) c+x d(c)+d(c) x .
$$

Since $d(c) \in Z(R)$, the identity $d(c x)=d(x) c+x d(c)$ follows, as required.

Now we prove a special case of our Theorem 1.1.

Lemma 2.3. Suppose that $R$ is a PI-ring. If $d: R \rightarrow Q(R)$ is an additive map such that $d\left(x^{n+1}\right)=\sum_{j=0}^{n} x^{n-j} d(x) x^{j}$ for all $x \in R$, then $d$ is a derivation.

Proof. By Posner's theorem [13, p. 57], $Z(R) \neq\{0\}, Q(R)$ is a simple Artinian algebra and $Q(R)=R C=R Z(R)^{-1}$. By Lemma 2.1, we see that (2.1) holds for all $x \in R$. By Lemma 2.2,

$$
d(c x)=c d(x)+d(c) x
$$

for all $x \in R$ and $c \in Z(R)$. Since $Q(R)=R C=R Z(R)^{-1}$, we can extend the map $d: R \rightarrow Q(R)$ to the map $\tilde{d}: Q(R) \rightarrow Q(R)$ defined by

$$
\tilde{d}\left(c^{-1} x\right):=c^{-2}(c d(x)-x d(c)) \quad \forall x \in R, \forall c \in Z(R) \backslash\{0\} .
$$

We claim that the expression for $\tilde{d}$ in (2.15) is well defined. Let $\alpha^{-1} x=\beta^{-1} y$, where $x, y \in R$ and $\alpha, \beta \in Z(R) \backslash\{0\}$. Since $\beta x=\alpha y$, we see by (2.14) that

$$
\beta d(x)-y d(\alpha)=\alpha d(y)-x d(\beta) .
$$

A direct computation proves that

$$
\beta^{2}(\alpha d(x)-x d(\alpha))=\alpha^{2}(\beta d(y)-y d(\beta)) .
$$

Therefore, $\tilde{d}\left(\alpha^{-1} x\right)=\tilde{d}\left(\beta^{-1} y\right)$, as asserted.

We need to prove that $\tilde{d}$ is also an additive map satisfying (1.1). The proof of the additivity of $\tilde{d}$ is straightforward. Since $d(c) \in Z(R)$ by Lemma 2.2, we can deduce from (2.15) that

$$
\begin{aligned}
\tilde{d}\left(\left(c^{-1} x\right)^{n+1}\right) & =c^{-2 n-2}\left(c^{n+1} d\left(x^{n+1}\right)-x^{n+1} d\left(c^{n+1}\right)\right) \\
& =c^{-n-1} \sum_{j=0}^{n} x^{n-j} d(x) x^{j}-(n+1) c^{-n-2} x^{n+1} d(c) \\
& =c^{-n-2} \sum_{j=0}^{n} x^{n-j} c d(x) x^{j}-c^{-n-2} \sum_{j=0}^{n} x^{n-j}(x d(c)) x^{j}
\end{aligned}
$$




$$
\begin{aligned}
& =\sum_{j=0}^{n}\left(c^{-1} x\right)^{n-j}\left(c^{-2}(c d(x)-x d(c))\right)\left(c^{-1} x\right)^{j} \\
& =\sum_{j=0}^{n}\left(c^{-1} x\right)^{n-j} \tilde{d}\left(c^{-1} x\right)\left(c^{-1} x\right)^{j},
\end{aligned}
$$

as asserted. Since $Q(R)$ has an identity, it follows from [7, Theorem 2] that $\tilde{d}$ is a derivation on $Q(R)$. In particular, $d$ is a derivation on $R$. This completes the proof.

Recall that Herstein [11, Theorem 3.1] proved that a Jordan derivation on a prime ring $R$ of characteristic other than 2 is a derivation. We remark that, by a trivial check, this result still holds if $d$ maps from $I$ to $R$. So, we state it here in the following form.

Theorem 2.4 (See [11, Theorem 3.1]). Suppose that $R$ is a prime ring and $\operatorname{char}(R) \neq 2$. If $d: I \rightarrow R$ is an additive map such that $d\left(x^{2}\right)=x d(x)+d(x) x$ for all $x \in I$, where $I$ is an ideal of $R$, then $d(x y)=x d(y)+d(x) y$ for all $x, y \in I$.

To prove Theorem 1.1, we also need some results from functional identities. For $x \in R$, let $\operatorname{deg}(x)$ be the minimal degree of $x$ over $C$ if $x$ is algebraic over $C$ and $\infty$ otherwise. For a subset $A$ of $R$, we define

$$
\operatorname{deg}(A)=\sup \{\operatorname{deg}(x) \mid x \in A\} .
$$

It is known that $\operatorname{deg}(I)=\operatorname{deg}(R)$ for any nonzero ideal $I$ of $R$ and that $\operatorname{deg}(R) \leq m$ for some positive integer $m$ if and only if $R$ is a PI-ring. We refer to [5] for more details on functional identities. Now we are ready to prove Theorem 1.1.

Proof of Theorem 1.1 First suppose that $R$ is a PI-ring. Then $I$ itself is also a prime PI-ring. By Posner's theorem [13, p. 57], $Z(R) \neq\{0\}, Q(R)$ is a simple Artinian algebra and $Q(R)=R C=R Z(R)^{-1}$. Moreover, it is well known that $Q(I)=Q(R)=R C$ in this case. So, we can regard $d: I \rightarrow R$ as $d: I \rightarrow Q(I)$. Hence, by Lemma 2.3, $d$ is a derivation on $I$.

Next suppose that $R$ is not a PI-ring. By Lemma 2.1,

$$
\sum_{j=0}^{n}\left((n-j) x^{2 n-2 j-1} T(x, x) x^{2 j}+(n-j) x^{2 j} T(x, x) x^{2 n-2 j-1}\right)=0
$$

for all $x \in I$, where

$$
T(x, y)=2^{-1}(d(x y)-d(x) y-x d(y)+d(y x)-d(y) x-y d(x))
$$

is a symmetric biadditive map. Since the sum of all of the coefficients is $n(n+1)$, which is not 0 , we can conclude by [1, Theorem 4.6] that $T$, given by

$$
T(x, x)=d\left(x^{2}\right)-d(x) x-x d(x),
$$

is a zero map. This means that $d$ is a Jordan derivation on $I$. So, $d$ is a derivation on $I$ by Theorem 2.4. This completes the proof. 


\section{An application}

As an application, we generalize Theorem 1.1 to generalized derivations. Let $I$ be an ideal of a ring $R$. An additive map $g: I \rightarrow R$ is called a generalized derivation on $I$ if there exists a derivation $d: I \rightarrow R$ such that $g(x y)=g(x) y+x d(y)$ for all $x, y \in I$. For example, if $g(x)=a x+d(x)$, where $a \in R$ and $d$ is a derivation on $R$, then $g$ is a generalized derivation on $R$.

An additive map $g$ is called a generalized Jordan derivation of $R$ if there exists a Jordan derivation $d$ such that $g\left(x^{2}\right)=g(x) x+x d(x)$ for all $x \in R$. A generalized derivation is, of course, a generalized Jordan derivation. By analogy with Herstein's theorem about Jordan derivations (see Theorem 2.4), Jing and Lu [14, Theorem 2.5] proved the converse for generalized Jordan derivations. Their theorem may be stated as follows. If $R$ is a prime ring of characteristic other than 2, and $g$ is a generalized Jordan derivation of $R$, then $g$ is a generalized derivation. Now we prove the following generalization.

CoRollary 3.1. Let $n$ be a positive integer. Suppose that $R$ is a prime ring whose characteristic is either 0 or a prime $p$ that is greater than $2 n$. Let I be a nonzero ideal of $R$. If $g: I \rightarrow R$ and $d: I \rightarrow R$ are additive maps such that

$$
d\left(x^{n+1}\right)=\sum_{j=0}^{n} x^{j} d(x) x^{n-j}, \quad g\left(x^{n+1}\right)=g(x) x^{n}+\sum_{j=1}^{n} x^{j} d(x) x^{n-j}
$$

for all $x \in I$, then $g$ is a generalized derivation on I and $d$ is the associated derivation of $g$.

Proof. By direct computation, we see that

$$
g\left(x^{n+1}\right)-d\left(x^{n+1}\right)=g(x) x^{n}-d(x) x^{n} .
$$

That is, $g-d$ is an additive map and

$$
(g-d)\left(x^{n+1}\right)=(g-d)(x) x^{n} \quad \forall x \in I .
$$

By [16, Theorem 3.8], there exists $a \in Q_{r}(R)$ such that $(g-d)(x)=a x$ for all $x \in I$. Moreover, $d$ is a derivation by Theorem 1.1. Hence, $g(x)=a x+d(x)$ is a generalized derivation on $I$, as asserted.

\section{Acknowledgements}

The author thanks Professor Tsiu-Kwen Lee of National Taiwan University for helpful comments and the referees for their useful suggestions and comments.

\section{References}

[1] K. I. Beidar, 'On functional identities and commuting additive mappings', Comm. Algebra 26 (1998), 1819-1850.

[2] K. I. Beidar, M. Brešar, M. A. Chebotar and W. S. Martindale 3rd, 'On Herstein's Lie map conjectures II', J. Algebra 238 (2001), 239-264. 
[3] K. I. Beidar, W. S. Martindale 3rd and A. V. Mikhalev, Rings with Generalized Identities (Marcel Dekker, New York-Basel-Hong Kong, 1996).

[4] M. Brešar, 'Jordan derivations on semiprime rings', Proc. Amer. Math. Soc. 104 (1988), 1003-1006.

[5] M. Brešar, 'Functional identities: a survey', Contemp. Math. 259 (1999), 93-109.

[6] M. Brešar and J. Vukman, 'Jordan derivations on prime rings', Bull. Aust. Math. Soc. 37 (1988), 321-322.

[7] D. Bridges and J. Bergen, 'On the derivation of $x^{n}$ in a ring', Proc. Amer. Math. Soc. 90 (1984), 25-29.

[8] C.-L. Chuang, 'GPIs having coefficients in Utumi quotient rings', Proc. Amer. Math. Soc. 103 (1988), 723-728.

[9] J. M. Cusack, 'Jordan derivations on rings', Proc. Amer. Math. Soc. 53 (1975), 321-324.

[10] M. Fošner and J. Vukman, 'On some functional equations in rings', Comm. Algebra 39 (2011), 2647-2658.

[11] I. N. Herstein, 'Jordan derivations of prime rings', Proc. Amer. Math. Soc. 8 (1957), 1104-1110.

[12] I. N. Herstein, Topics in Ring Theory (The University of Chicago Press, Chicago-London, 1969).

[13] N. Jacobson, PI-Algebras: An Introduction, Lecture Notes in Mathematics, 441 (Springer, Berlin, 1975).

[14] W. Jing and S. Lu, 'Generalized Jordan derivations on prime rings and standard operator algebras', Taiwanese J. Math. 7 (2003), 605-613.

[15] V. K. Kharchenko, 'Differential identities of prime rings', Algebra Logika 17 (1978), 220-238 Engl. Transl., Algebra Logic 17 (1978), 154-168.

[16] T.-K. Lee and K.-S. Liu, 'Certain additive maps on $m$-power closed Lie ideals', Monatsh. Math. 164 (2011), 287-298.

[17] W. S. Martindale 3rd, 'Prime rings satisfying a generalized polynomial identity', J. Algebra 12 (1969), 576-584.

[18] J. Vukman and I. Kosi-Ulbl, 'A note on derivations in semiprime rings', Int. J. Math. Math. Sci. 20 (2005), 3347-3350.

KUN-SHAN LIU, Department of Mathematics, National Taiwan University, Taipei 106, Taiwan e-mail: kunshanliu@ntu.edu.tw 